\title{
Promiscuous Pathogenicity Islands and Phylogeny of Pathogenic Streptomyces spp.
}

\author{
Yucheng Zhang, ${ }^{1}$ Dawn R. D. Bignell, ${ }^{2}$ Ran Zuo, ${ }^{3}$ Qiurong Fan, ${ }^{4}$ Jose C. Huguet-Tapia, ${ }^{1}$ Yousong Ding, ${ }^{3}$ \\ and Rosemary Loria ${ }^{1}$ \\ ${ }^{1}$ Department of Plant Pathology, University of Florida, Gainesville, Florida, U.S.A.; ${ }^{2}$ Department of Biology, Memorial University \\ of Newfoundland, St. John's, Newfoundland and Labrador, Canada; ${ }^{3}$ Department of Medicinal Chemistry, University of Florida, \\ Gainesville, Florida, U.S.A.; and ${ }^{4}$ College of Plant Protection, Fujian Agriculture and Forestry University, Fuzhou, Fujian, China
}

Accepted 5 July 2016.

\begin{abstract}
Approximately 10 Streptomyces species cause disease on underground plant structures. The most economically important of these is potato scab, and the most studied of these pathogens is Streptomyces scabiei (syn. S. scabies). The main pathogenicity determinant of scab-causing Streptomyces species is a nitrated diketopiperazine, known as thaxtomin A (ThxA). In the pathogenic species Streptomyces turgidiscabies, ThxA biosynthetic genes reside on a mobile pathogenicity island (PAI). However, the mobilization of PAIs in other Streptomyces species remains uncharacterized. Here, we investigated the mobilization of the PAI of $S$. scabiei 87-22. Based on whole genome sequences, we inferred the evolutionary relationships of pathogenic Streptomyces species and discovered that Streptomyces sp. strain 96-12, a novel pathogenic species isolated from potatoes in Egypt, was phylogenetically grouped with nonpathogenic species rather than with known pathogenic species. We also found that Streptomyces sp. strain 96-12 contains a PAI that is almost identical to the PAI in S. scabiei 87-22, despite significant differences in their genome sequences. This suggested direct or indirect in vivo mobilization of the PAI between $S$. scabiei and nonpathogenic Streptomyces species. To test whether the S. scabiei 87-22 PAI could, indeed, be mobilized, S. scabiei 87-22 deletion mutants containing antibiotic resistance markers in the PAI were mated with Streptomyces diastatochromogenes, a nonpathogenic species. The PAI of $S$. scabiei was site-specifically inserted into the aviXI gene of $S$. diastatochromogenes and conferred pathogenicity in radish seedling assays. Our results demonstrated that $S$. scabiei, the earliest described Streptomyces pathogen, could be the source of a PAI responsible for the emergence of novel pathogenic species.
\end{abstract}

Streptomyces spp. are gram-positive, filamentous actinobacteria with high $\mathrm{G}+\mathrm{C}$ content genomes. They are prolific producers of biologically active, secondary metabolites, including two-thirds of currently used antibiotics (Bentley et al. 2002). A small number of these saprophytic bacteria are plant pathogens (Loria et al. 2006). The best characterized of these are Streptomyces scabiei, Streptomyces acidiscabies, Streptomyces turgidiscabies, and Streptomyces ipomoeae (Loria et al. 2008). Mobilization of pathogenicity

Corresponding author: Rosemary Loria; E-mail: rloria@ufl.edu

*The $e$-Xtra logo stands for "electronic extra" and indicates that three supplementary figures and four supplementary tables are published online.

(C) 2016 The American Phytopathological Society islands (PAIs) is thought to be a driving force in the evolution of plant-pathogenic streptomycetes (Loria et al. 2006). Bioinformatic analyses of the genome sequences of S. scabiei 87-22, S. acidiscabies 84-104 (Huguet-Tapia and Loria 2012), S. turgidiscabies Car8 (Huguet-Tapia et al. 2014), and S. ipomoeae 91-03 (Guan et al. 2012; Huguet-Tapia et al. 2016) revealed related but not identical PAIs.

The 674-kb PAI discovered in S. turgidiscabies (HuguetTapia et al. 2014; Kers et al. 2005) was the first PAI described in any gram-positive plant pathogen. This PAI carries genes encoding four pathogenicity and virulence factors: the biosynthetic pathway for thaxtomin A (ThxA), a functional tomatinase (TomA), a small, secreted protein (Nec1), and a cytokinin biosynthetic pathway (Fas) (Huguet-Tapia et al. 2014). ThxA is a nitrated 2,5-diketopiperazine synthesized via two nonribosomal peptide synthetases (NRPS) encoded by the $t x t A$ and $t x t B$ genes, a P450 monooxygenase (TxtC), a nitric oxide synthase (TxtD), and a novel cytochrome P450 (TxtE) that site-specifically nitrates tryptophan prior to cyclization (Bignell et al. 2014). A 65-amino acid protein encoded by $t x t H$ is a predicted MbtH-like family protein and may be important in regulating NRPS activity (Herbst et al. 2013; Stegmann et al. 2006). The expression of $t x t H$ was shown to be increased under thaxtomin-inducing conditions (Bignell et al. 2010a). However, its role in ThxA production is the subject of ongoing research. ThxA inhibits cellulose biosynthesis in plant cell walls, leading to abnormal cell expansion and cell death (Loria et al. 2008). TomA and Nec1 are not essential for pathogenicity but may play roles in the infection process, possibly by suppressing plant defenses (Joshi et al. 2007; Seipke and Loria 2008). These four virulence factors also exist in S. scabiei and S. acidiscabies, but they are separated in two remote chromosomal regions, designated the toxicogenic region (TR) and the colonization region (CR) (Lerat et al. 2009). The TR contains the ThxA biosynthetic gene cluster, while the $\mathrm{CR}$ includes necl and tomA. In addition to these genes, the PAI in $S$. turgidiscabies also contains the fas operon with genes that are homologous to those in Rhodococcus fascians (Joshi and Loria 2007); Fas proteins mediate the biosynthesis and modification of a mix of cytokinins, which contribute to virulence phenotypes in both pathogens (Pertry et al. 2010).

Dissecting the evolution of PAIs in Streptomyces spp. requires an understanding of the phylogenetic relationships among pathogenic species and related nonpathogenic species. The analysis of $16 \mathrm{~S}$ ribosomal RNA (rRNA) sequences has become the standard approach for determining evolutionary relationships in this genus (Kreuze et al. 1999), but highly similar 16S rRNA sequences among Streptomyces species limits the usefulness of this approach (Labeda et al. 2012). 
Genes encoding DNA topoisomerase, ribosomal protein S1, SecY, and GTPase were used for phylogenetic analysis of Streptomyces spp., but no single gene yielded a resolved phylogeny (Alam et al. 2010). To address this issue, multilocus sequence analysis was developed (Guo et al. 2008); multiple internal fragments of ATP synthase F1 ( $\beta$-subunit; atpD), DNA gyrase $(\beta$-subunit; $g y r B)$, recombinase A $(\operatorname{rec} A)$, RNA polymerase ( $\beta$-subunit; rpoB), and tryptophan synthase ( $\beta$-subunit; $\operatorname{trp} B$ ) have successfully resolved the evolutionary relationships of some closely related Streptomyces species (Guo et al. 2008). However, in other bacterial genera, concatenation of hundreds of selected single-copy genes from microbial genomes as a single dataset is now possible, and comparative analyses of these datasets have been used to reconstruct highly resolved evolutionary relationships (Salichos and Rokas 2013).

Herein, we investigated the evolutionary history of scabcausing pathogenic streptomycetes. Specifically, we sequenced the genomes of ten phytopathogenic Streptomyces isolates from various geographic origins. We performed a phylogenomic and systematic analysis of their genomes along with 100 Streptomyces genomes deposited in the GenBank database; fortunately, among the 100 genomes, nine were recently sequenced Streptomyces phytopathogens. Our studies strongly indicated that the phytopathogen Streptomyces sp. strain 96-12 acquired the PAI from S. scabiei directly or indirectly, likely in an agricultural environment. Remarkably, we further demonstrated that the $S$. scabiei PAI can transfer and site-specifically integrate into the chromosome of saprophytic species under laboratory conditions. Acquisition of the PAI conferred a pathogenic phenotype on saprophytic species. These results confirm the mobility of the S. scabiei PAI to nonpathogenic species, both in vivo and in vitro, and lay the groundwork to further understand and predict the pathogenic development of plant pathogens.

\section{RESULTS}

\section{Characterization of pathogenic Streptomyces spp.}

We sequenced the genomes of 10 isolates of plant-pathogenic streptomycetes from different locations and the genome of Streptomyces diastatochromogenes ATCC 12309, a nonpathogenic isolate. To validate the pathogenicity of selected plant pathogens, we tested their ability to cause disease symptoms on radish seedlings. Scab-causing Streptomyces species cause tissue swelling and necrosis and severe root and shoot stunting, most likely due to the production of ThxA (Loria et al. 1997). All tested plant-pathogenic isolates induced similar disease symptoms on radish seedlings, following infection (Supplementary Fig. S1A).

ThxA inhibits plant cellulose synthesis in the parts per million range (Fry and Loria 2002). Its superior potency has attracted industrial interests in developing ThxA as a natural and biodegradable herbicide to control weed growth (King et al. 2001). Cellobiose, a strong elicitor of ThxA production (Johnson et al. 2007), can significantly induce ThxA production. However, marketing ThxA as a herbicide is not profitable, due to the high cost of cellobiose. Therefore, pathogenic isolates with the ability to produce a large amount of ThxA without cellobiose can be used as the industrial strain (Francis et al. 2015). We used high-pressure liquid chromatography (HPLC) analysis to quantify ThxA production levels of different pathogenic Streptomyces isolates cultured in oat bran broth (OBB), an undefined complex medium known to induce ThxA biosynthesis (Wach et al. 2007). Our assay also included three well-studied pathogenic isolates, namely S. scabiei 87-22, $S$. acidiscabies 84-104, and S. turgidiscabies Car8. The pathogenic Streptomyces isolates produced ThxA at different levels. S. acidiscabies 84-104 produced the highest level of ThxA, followed by $S$. turgidiscabies Car8 and S. scabiei 96-08. All other tested isolates produced a significantly lower level of ThxA. ThxA was undetectable in the extracts of Streptomyces europaeiscabiei 96-14 and Streptomyces sp. strain 96-12 cultured in OBB. This result was unexpected, but when OBB was supplemented with $1 \%$ cellobiose $(\mathrm{OBBc})$, ThxA production levels of all strains were increased and ThxA production by S. europaeiscabiei 96-14 and Streptomyces sp. strain 96-12 was also detected (data not shown), confirming their ability to produce ThxA.

\section{Phylogenomic analysis of the Streptomyces genus.}

To accurately infer the phylogenetic relationships of pathogenic Streptomyces species in the genus, we performed a comprehensive phylogenomic analysis using 111 Streptomyces genomes (Supplementary Tables S1 and S2). We first identified 542 single-copy ortholog groups that are shared among all 111 Streptomyces isolates as well as two outgroups (Streptacidiphilus albus JL83 and Kitasatospora setae NBRC 14216), using OrthoMCL v1.4 (Li et al. 2003). The nucleotide sequence alignments of the 542 ortholog groups were filtered by removing poorly aligned sites (less than $50 \%$ of the original alignment length), resulting in the exclusion of 109 ortholog groups. A concatenated alignment was made from the remaining 433 genes and was used to infer the phylogenetic relationships of the 111 Streptomyces isolates by maximum likelihood (ML). The ML tree showed strong bootstraps in almost all branches, with only a few nodes receiving less than $70 \%$ bootstrap support (Fig. 1).

S. scabiei isolates from different geographical regions were clustered closely together in the phylogram. Interestingly, Streptomyces stelliscabiei was phylogenetically closer to the nonpathogenic species Streptomyces bottropensis than to other pathogenic species, including S. scabiei. Our analysis demonstrated that S. scabiei and S. bottropensis were phylogenetically distant from $S$. diastatochromogenes (Fig. 1), although they were previously thought of as closely related species and were assigned to the Diastatochromogenes cluster (Elesawy and Szabo 1979; Healy and Lambert 1991). S. ipomoeae, which produces a less-modified thaxtomin derivative (thaxtomin $\mathrm{C}$ ) (Guan et al. 2012), formed a separate clade from the cluster containing pathogenic S. scabiei, S. europaeiscabiei, and S. stelliscabiei in our analysis. Streptomyces sp. strain 96-12 was originally isolated from potatoes in Egypt and was classified as $S$. scabiei. Interestingly, our phylogenomic analysis grouped it with the nonpathogenic strain Streptomyces galbus KCCM 41354 rather than with other phytopathogenic species. This result suggested that Streptomyces sp. strain 96-12 may be a recently emerged, novel pathogenic species. S. acidiscabies 84-104 and S. turgidiscabies Car8 were also phylogenetically grouped with nonpathogenic isolates, substantiating the notions that these two pathogenic species are emergent pathogens (Huguet-Tapia and Loria 2012; Huguet-Tapia et al. 2011).

\section{In silico DNA-DNA hybridization (isDDH) and average nucleotide identity (ANI) comparison of plant-pathogenic Streptomyces isolates.}

To further define the evolutionary relationships of pathogenic Streptomyces isolates, we used two different in silico approaches, isDDH and ANI, to assess genome similarity. Wetlab DDH was a cornerstone of bacterial species determination, but it is tedious and difficult to standardize among laboratories (Achtman and Wagner 2008). In contrast, isDDH data are reproducible and straightforward when genome sequences are available (Goris et al. 2007). The ANI of all genes shared by isolates provides a good estimate of evolutionary distance (Colston et al. 2014; Richter and Rosselló-Móra 2009; Zhang 
and Qiu 2015; 2016; Zhang et al. 2016). Generally, the 95 to $96 \%$ ANI value corresponds to the $70 \%$ DDH standard; both values have been used as cutoffs in species discrimination (Goris et al. 2007).
Our analysis demonstrated that the ANI and isDDH values of each strain pair (Fig. 2) correlated well with their phylogenetic relationships in the ML tree (Fig. 1), and strains in the same phylogenetic clade also showed high ANI and isDDH values.

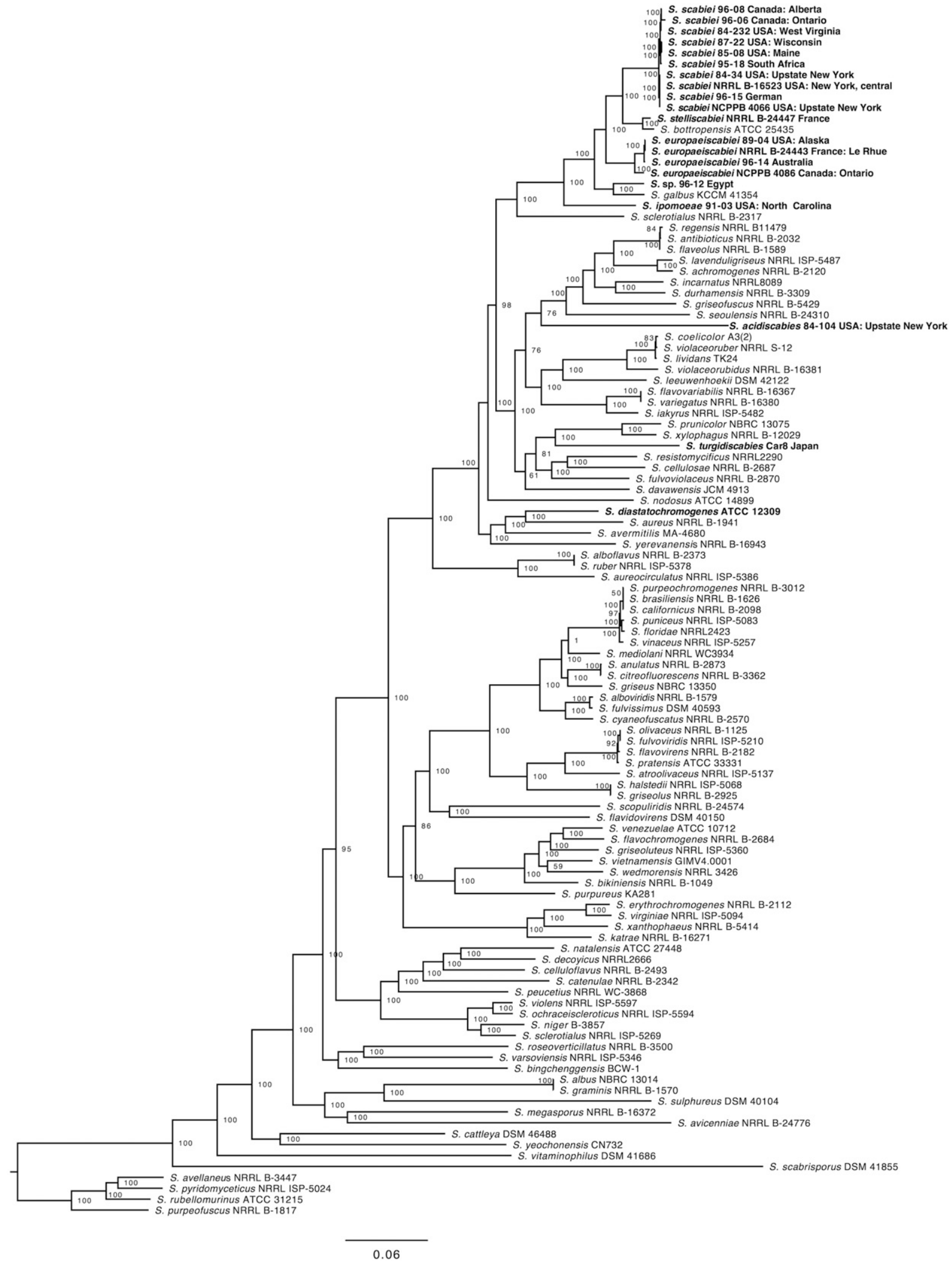

Fig. 1. Phylogenetic reconstruction of the genus Streptomyces based on the concatenation of 433 single-copy gene clusters from 111 Streptomyces genomes, with the maximum likelihood algorithm RAxML. A total of 1,000 bootstrap replicates were computed. Numbers above branches are bootstrap percentages. Streptacidiphilus albus JL83 and Kitasatospora setae NBRC 14216 were selected as outgroups (not shown on tree). 
For example, ANI and isDDH values (Fig. 2) as well as the core genome-based phylogeny (Fig. 1) all indicated Streptomyces sp. strain 96-12 is distinct from S. bottropensis, S. scabiei, and S. europaeiscabiei, although its 16S rRNA sequence is $100 \%$ identical to $S$. bottropensis and $99 \%$ identical to the latter two strains (data not shown). In addition, S. europaeiscabiei NCPPB 4086 was named $S$. scabiei NCPPB 4086 when deposited in GenBank (GenBank accession number GCA_000738695.1), but our analysis unambiguously classified it as a $S$. europaeiscabiei isolate. The isDDH values between $S$. europaeiscabiei NCPPB 4086 and other S. europaeiscabiei isolates were approximately $73 \%$. Given that an isDDH value of $79 \%$ is usually used as the lower limit in classifying subspecies (Meier-Kolthoff et al. 2013), our work further indicated that S. europaeiscabiei NCPPB 4086 and other $S$. europaeiscabiei isolates may belong to two different subspecies. Furthermore, S. bottropensis and S. stelliscabiei shared high ANI (97\%) and isDDH (75\%) values. This result agreed well with the conclusion of the phylogenomic analysis (Fig. 1 ) and suggested that S. stelliscabiei and S. bottropensis should be classified as subspecies of a single species. Further comparative analysis of closely related pathogenic and nonpathogenic strains may be useful in identifying virulence genes.

\section{Streptomyces pathogens contained different versions of PAIs.}

To further understand the emergence and evolution of plant pathogenicity, we focused on comparative genomic analysis of PAIs in various Streptomyces species (Fig. 3). S. turgidiscabies Car8 contains a uniquely large PAI of $674 \mathrm{~kb}$, which includes the ThxA biosynthetic cluster, the virulence genes necl and tomA, and a fas operon (Huguet-Tapia et al. 2011). In contrast, virulence genes in the PAIs of other pathogenic species are separated into two distinct regions (TR and CR) and the fas operon is missing (Fig. 3). One internal and two flanking attachment (att) sites of the $S$. scabiei TR further separate its TR into two subregions, designated TR1 and TR2 (Chapleau et al. 2016). The 20-kb TR1 region contains the ThxA biosynthetic cluster, and the 157-kb TR2 region includes putative integrative

\begin{tabular}{|c|c|c|c|c|c|c|c|c|c|c|c|c|c|c|c|c|c|c|c|c|c|}
\hline $\begin{array}{c}=\mathrm{ANI} \geq 99 \% \\
\text { or } \\
\text { is } \mathrm{DDH}+\text { C. I. } \geq 79 \% \\
=\mathrm{ANI} \geq 96 \% \text { and }<99 \% \\
\text { or } \\
\text { is } \mathrm{DDH}+\text { C. } \mathrm{I} . \geq 70 \% \text { and }<79 \%\end{array}$ & 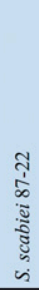 & 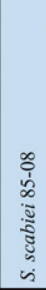 & 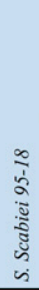 & 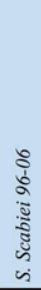 & 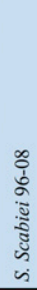 & 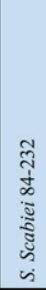 & 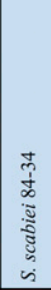 & 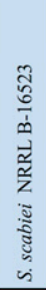 & 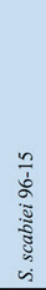 & 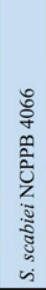 & 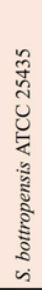 & 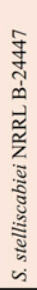 & 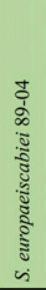 & 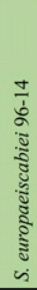 & 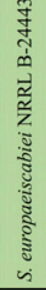 & 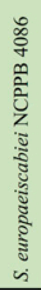 & $\begin{array}{l}\frac{\sim}{b} \\
\text { oे } \\
\text { के } \\
\text { मे }\end{array}$ & 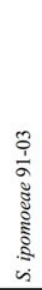 & 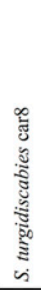 & 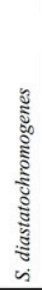 & 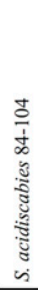 \\
\hline S. scabiei $87-22$ & & 100 & 98 & 98 & 93 & 97 & 97 & 97 & 98 & 97 & 46 & 48 & 42 & 42 & 42 & 42 & 40 & 34 & 29 & 29 & 28 \\
\hline S. scabiei $85-08$ & 99 & & 99 & 99 & 99 & 99 & 98 & 97 & 99 & 97 & 47 & 49 & 43 & 43 & 43 & 42 & 40 & 34 & 29 & 29 & 28 \\
\hline S. scabiei $95-18$ & 99 & 99 & & 99 & 94 & 99 & 99 & 98 & 98 & 97 & 47 & 49 & 43 & 43 & 43 & 42 & 41 & 34 & 30 & 29 & 28 \\
\hline S. scabiei $96-06$ & 99 & 99 & 99 & & 98 & 100 & 100 & 99 & 99 & 99 & 47 & 49 & 43 & 43 & 43 & 42 & 41 & 34 & 30 & 29 & 28 \\
\hline S. scabiei $96-08$ & 99 & 99 & 99 & 99 & & 96 & 95 & 94 & 94 & 93 & 47 & 48 & 45 & 45 & 46 & 45 & 41 & 34 & 30 & 29 & 28 \\
\hline S. scabiei $84-232$ & 99 & 99 & 99 & 99 & 99 & & 99 & 98 & 99 & 98 & 47 & 48 & 43 & 43 & 43 & 42 & 41 & 34 & 29 & 29 & 28 \\
\hline S. scabiei $84-34$ & 99 & 99 & 99 & 99 & 99 & 99 & & 100 & 100 & 100 & 47 & 49 & 43 & 43 & 43 & 42 & 41 & 34 & 29 & 29 & 28 \\
\hline S. scabiei NRRL B-16523 & 99 & 99 & 99 & 99 & 99 & 99 & 100 & & 100 & 100 & 47 & 49 & 43 & 43 & 43 & 42 & 40 & 34 & 29 & 29 & 28 \\
\hline S. scabiei $96-15$ & 99 & 99 & 99 & 99 & 99 & 99 & 100 & 100 & & 100 & 47 & 50 & 43 & 43 & 43 & 43 & 41 & 34 & 29 & 29 & 28 \\
\hline S. scabiei NCPPB 4066 & 99 & 99 & 99 & 99 & 99 & 99 & 100 & 100 & 100 & & 46 & 49 & 42 & 42 & 43 & 42 & 40 & 34 & 29 & 29 & 28 \\
\hline S. bottropensis ATCC 25435 & 92 & 92 & 92 & 92 & 92 & 92 & 92 & 92 & 92 & 92 & & 75 & 45 & 45 & 45 & 44 & 40 & 34 & 28 & 29 & 27 \\
\hline S. stelliscabiei NRRL B-24447 & 92 & 92 & 92 & 92 & 92 & 92 & 92 & 92 & 93 & 92 & 97 & & 46 & 46 & 46 & 45 & 41 & 35 & 29 & 29 & 28 \\
\hline S. europaeiscabiei $89-04$ & 91 & 91 & 91 & 91 & 91 & 91 & 91 & 91 & 91 & 91 & 92 & 92 & & 98 & 100 & 72 & 41 & 35 & 29 & 29 & 28 \\
\hline S. europaeiscabiei $96-14$ & 91 & 91 & 91 & 91 & 91 & 91 & 91 & 91 & 91 & 91 & 91 & 91 & 99 & & 98 & 72 & 41 & 35 & 29 & 29 & 28 \\
\hline S. europaeiscabiei NRRL B- 24443 & 91 & 91 & 91 & 91 & 91 & 91 & 91 & 91 & 91 & 91 & 92 & 92 & 100 & 99 & & 73 & 41 & 35 & 29 & 29 & 28 \\
\hline S. europaeiscabiei NCPPB 4086 & 91 & 91 & 91 & 91 & 91 & 91 & 91 & 91 & 91 & 91 & 91 & 91 & 97 & 96 & 96 & & 41 & 35 & 29 & 29 & 27 \\
\hline S. sp. $96-12$ & 90 & 90 & 90 & 90 & 90 & 90 & 90 & 90 & 90 & 90 & 90 & 90 & 91 & 90 & 90 & 90 & & 35 & 29 & 29 & 27 \\
\hline S. ipomoeae $91-03$ & 88 & 88 & 88 & 88 & 88 & 88 & 88 & 88 & 88 & 88 & 88 & 88 & 89 & 88 & 88 & 88 & 88 & & 29 & 30 & 34 \\
\hline S. turgidiscabies car8 & 87 & 87 & 87 & 87 & 87 & 87 & 87 & 87 & 87 & 87 & 86 & 87 & 87 & 87 & 87 & 86 & 86 & 86 & & 29 & 27 \\
\hline S. diastatochromogenes & 86 & 86 & 86 & 86 & 86 & 86 & 86 & 86 & 86 & 86 & 86 & 86 & 86 & 86 & 86 & 86 & 86 & 86 & 86 & & 27 \\
\hline S. acidiscabies $84-104$ & 86 & 86 & 86 & 86 & 86 & 86 & 86 & 86 & 86 & 86 & 86 & 86 & 86 & 86 & 86 & 85 & 86 & 86 & 86 & 85 & \\
\hline
\end{tabular}

Fig. 2. Average nucleotide identity (ANI) and in silico DNA-DNA hybridization (isDDH) values of plant-pathogenic and closely related nonpathogenic Streptomyces isolates. The lower triangle displays ANI values, and the upper triangle displays isDDH values. Displayed isDDH values are point estimates plus the $95 \%$ model-based confidence intervals. Boxes with ANI $\geq 99 \%$ or isDDH $\geq 79 \%$ are colored red, and boxes with ANI $\geq 96$ and $<99 \%$ or isDDH $\geq 70$ and $<79 \%$ are colored orange. Isolates belonging to the same species are marked with the same color. 
and conjugative elements (ICEs) (Chapleau et al. 2016). Our analysis demonstrated that all pathogenic isolates possess the TR1 region, indicating the importance of ThxA in disease development. The TR2 region is conserved only in S. scabiei isolates and Streptomyces sp. strain 96-12. Our high-resolution evolutionary analysis revealed that Streptomyces sp. strain 9612 was phylogenetically separated from S. scabiei (Figs. 1 and 2). The same organization and contents of PAIs between Streptomyces sp. strain 96-12 and S. scabiei suggested an in vivo mobilization of the $S$. scabiei PAI. The TR of S. stelliscabiei NRRL B-24447 contains genes encoding the integrase and the recombination directionality factor that are essential for integration and excision (Wozniak and Waldor 2010), but we failed to identify the complete TR2 region due to the draft genome's fragmentation (Fig. 3). Furthermore, the S. stelliscabiei NRRL B-24447 integrase only had $83 \%$ sequence identity with the integrase of S. scabiei 87-22 at the amino acid level (data not shown). The boundaries and mobilization mechanism of S. stelliscabiei NRRL B-24447 TR will require further research.

The CR containing virulence genes necl and tomA is missing in S. europaeiscabiei NCPPB 4086 and Streptomyces sp. strain 96-12. Since the CR is $4.9 \mathrm{Mb}$ away from the TR, these two regions may have been transferred into pathogenic Streptomyces genomes via different horizontal gene transfer (HGT) events. In S. turgidiscabies Car8, the circular structure of the $\mathrm{CR}$ from site-specific recombination at two flanking att sites has been detected (Huguet-Tapia et al. 2014). However, the mobilization ability of the $\mathrm{CR}$, itself, requires further investigation. S. europaeiscabiei NCPPB 4086 and Streptomyces sp. strain 9612 may have lost the CR during evolution, but it is also possible that these two isolates have never acquired this region.
ThxA phylogeny contradicts the core-genome phylogeny.

Almost all scab-causing Streptomyces species produce ThxA, and the transfer of the TR containing the characteristic ThxA biosynthetic gene cluster is likely to be the genetic basis for emergence of new pathogenic Streptomyces species. Phylogenetic analysis of the ThxA biosynthetic genes may thus infer the mechanism of TR mobilization and its evolution. To test this, we constructed a phylogenetic tree of selected pathogens, using their $t x t A, t x t B, t x t C, t x t D, t x t E$, and $t x t R$ genes (Supplementary Fig. S2). In this analysis, S. acidiscabies 84-104, all S. scabiei isolates, and Streptomyces sp. strain 96-12 were closely grouped, indicating the high similarity of their ThxA clusters (Fig. 4). In contrast, S. acidiscabies 84-104, Streptomyces sp. strain 96-12 and $S$. scabiei were distantly related in the core-genome tree (Fig. 4). The high similarity of the ThxA clusters in these three phylogenetically distant pathogenic taxa ( $S$. acidiscabies, Streptomyces sp. strain 96-12, and S. scabiei) strongly suggested recent HGT.

\section{The S. scabiei PAI was site-specifically mobilized into the chromosome of nonpathogenic species.}

Our bioinformatic analyses suggested the direct or indirect in vivo mobilization of a PAI between Streptomyces sp. strain 9612 and S. scabiei (Fig. 4). In all S. scabiei strains and in Streptomyces sp. strain 96-12, the whole TR is integrated at the $3^{\prime}$ end of an aviX1 gene (SCAB_33441, encoding a putative ATP/GTP binding protein) (Fig. 3). The same integration occurred in S. acidiscabies and S. europaeiscabiei, although they only acquired the TR1 region (Fig. 3). These results suggest that the $3^{\prime}$ end of the aviX1 gene of Streptomyces species is a common location for site-specific integration of the TR.
S. scabiei strains

S. acidiscabies $84-104$

S. europaeiscabiei $89-04$

S. europaeiscabiei $96-14$

S. europaeiscabiei NCPPB 4086

S. stelliscabiei NRRL B-24447

S. turgidiscabies Car8

S. sp. $96-12$
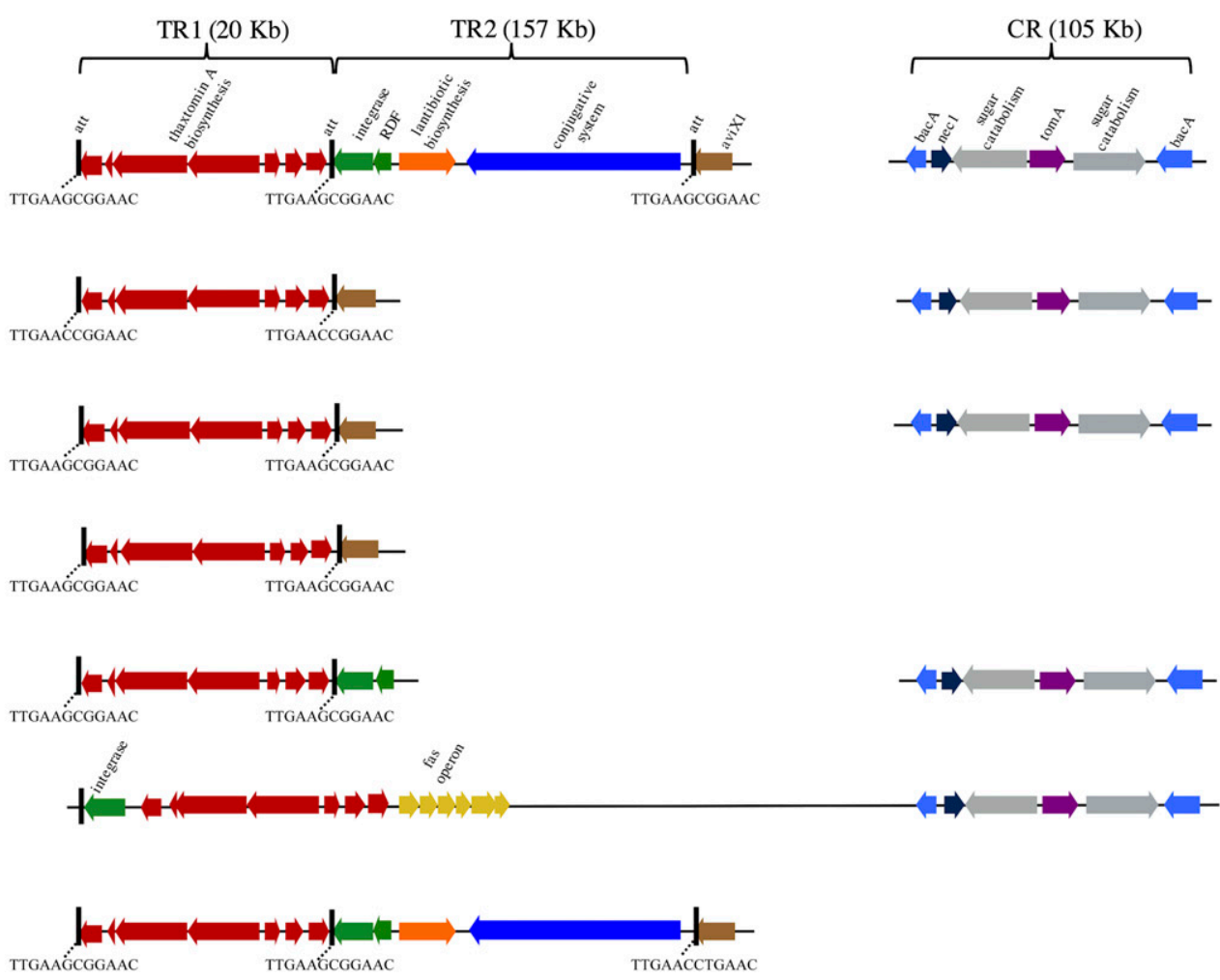

Fig. 3. Schematic genetic organization of pathogenicity islands from different plant-pathogenic Streptomyces species. $\mathrm{TR}=$ the toxicogenic region, $\mathrm{CR}=$ the colonization region. Arrowed boxes represent the location and orientation of the open reading frames of thaxtomin A biosynthetic genes (red), integrase and recombination directionality factor (RDF) (green), lantibiotic biosynthetic genes (orange), conjugative genes (blue), aviX1 (brown), bacA (light blue), necl (black), tomA (purple), sugar catabolism clusters (gray), and fas operon (yellow). The sequences of attachment (att) sites at the junctions for TR1, TR2, and TR are also shown. 
We aimed to further understand PAI mobilization and evolution by investigating the transfer of pathogenicity genes from S. scabiei to saprophytes in vitro. We first created two $S$. scabiei 87-22 deletion mutants, one with the apramycin resistance $\left(\operatorname{apr}^{\mathrm{R}}\right)$ marker inserted in its TR1 [S. scabiei $\left.\Delta t x t H\left(\operatorname{apr}^{\mathrm{R}}\right)\right]$ and the other with the hygromycin $\mathrm{B}$ resistance $\left(\right.$ hyg ${ }^{\mathrm{R}}$ ) marker inserted in its TR2 (S. scabiei $\left.\triangle \operatorname{lan} A B\left[\mathrm{hyg}^{\mathrm{R}}\right]\right)$. In mating experiments, these mutants served as the PAI donors, while naturally streptomycin-resistant $\left(\mathrm{str}^{\mathrm{R}}\right) \mathrm{S}$. diastatochromogenes, which is a nonpathogenic species, was used as the recipient strain (Fig. 5A). Following selection with apramycin or hygromycin B, together with streptomycin, a number of $S$. diastatochromogenes mutants were isolated. We found that the aviX1 gene of S. diastatochromogenes shared $93 \%$ identity with S. scabiei aviX1 (SCAB_33441) at the nucleic acid level (data not shown) and an identical att site (TTGAAGCGGAAC) (Fig. 5B). We mapped the mobilized PAI in multiantibioticresistant $S$. diastatochromogenes strains; the site-specific integration of the $S$. scabiei PAI into $S$. diastatochromogenes was examined using polymerase chain reaction (PCR) primers flanking the att site at the $3^{\prime}$ end of the $S$. diastatochromogenes aviX1 gene and other primers within TR1 or TR2 (Supplementary Fig. S3). We detected the integration of different S. scabiei TR regions into the att site of the $S$. diastatochromogenes aviX1 gene (Fig. 5C). Mating of S. scabiei $\triangle$ lanAB with $S$. diastatochromogenes resulted in the acquisition of TR2 alone or of the complete TR element by $S$. diastatochromogenes; however, all transconjugants from the mating of S. scabiei $\Delta t x t H$ with $S$. diastatochromogenes acquired the entire TR element but not TR1 alone. These observations suggested that the ICEs of the TR2 region are important to the mobilization and site-specific integration of TR2 or the entire TR region into a new host chromosome. In contrast, the TR 1 region carrying the ThxA biosynthetic cluster is not mobile alone and its transfer depends on the ICEs in the TR2 region.

The frequency of TR transfer, as estimated by the acquisition of TR2 alone or the entire TR by $S$. diastatochromogenes, using S. scabiei $\triangle$ lan $A B$ as the donor, is $1.1 \times 10^{-3} \pm 0.3 \times 10^{-3}$ of transconjugants per CFU of donor cells. Out of the 20 randomly selected transconjugants, $18(90 \%)$ were detected by PCR to contain TR2 alone, with only two transconjugants (10\%) carrying the entire TR (TR1 + TR2).

\section{PAI acquisition conferred a pathogenic phenotype} in S. diastatochromogenes transconjugants.

To determine the biological consequences of PAI transfer, we characterized $S$. diastatochromogenes transconjugants carrying different versions of $S$. scabiei-type PAI by determining their production of ThxA in $\mathrm{OBB}$ and $\mathrm{OBBc}$ and evaluating their pathogenicity with radish seedlings. $S$. diastatochromogenes transconjugants with TR2 $2^{\Delta l a n A B}$ alone produced an undetectable level of ThxA (data not shown) and were not pathogenic on radish seedlings (Fig. 5D). On the other hand, S. diastatochromogenes transconjugants that acquired the entire $\mathrm{TR}^{\triangle l a n A B}$ produced ThxA (Fig. 5E) and were pathogenic (Fig. 5D). Interestingly, the ThxA production level of these transconjugants was significantly higher than that of S. scabiei 87-22 in both OBB and OBBc. $S$. diastatochromogenes transconjugants carrying $\mathrm{TR}^{\Delta t x t H}$ in $\mathrm{OBB}$ and OBBc produced trace amounts of ThxA and two new metabolites with a distinctive yellow appearance (data not shown). HPLC and liquid chromatography-mass spectrometry (LC-MS) analysis identified the new compounds as thaxtomin D and monohydroxylated thaxtomin $\mathrm{D}$, presumably thaxtomin $\mathrm{B}$ (data not shown). The production of these two metabolites may be the result of insertion of the deletion cassette upstream of $t x t C$, which encodes a P450 monooxygenase responsible for converting thaxtomin D into ThxA (Healy et al. 2002). As expected, $S$. diastatochromogenes transconjugants carrying $\mathrm{TR}^{\Delta t x t H}$ were not pathogenic in the radish seedlings assays (Fig. 5D). Nonetheless, these results clearly demonstrated that the TR of S. scabiei-type PAI conferred pathogenicity to a nonpathogenic Streptomyces species.

\section{DISCUSSION}

It has been suggested that the S. scabiei TR has the capacity to mobilize to saprophytic Streptomyces isolates (Chapleau et al. 2016), similar to what has been observed with the S. turgidiscabies PAI (Kers et al. 2005), but this had not been confirmed. Here, we inserted two different antibiotic resistance markers into the $S$. scabiei TR1 and TR2 regions and monitored their mobilization and site-specific integration into the chromosome of a nonpathogenic species. We found that, although TR1, TR2, and the entire TR can be excised from the $S$. scabiei chromosome, only TR 2 or the entire TR was transferred into the recipient genome. Accordingly, recipients became pathogenic only after acquring the entire TR (Fig. 5). These results suggested that TR2 is required for mobilization and TR1 is required for pathogenicity and, more importantly, they are both involved in the emergence of novel plant-pathogenic Streptomyces species in agricultural systems. Furthermore, our phylogenomic and in silico genome similarity analyses (ANI and isDDH) suggested that the TR of Streptomyces sp. strain 96-12 may have been acquired, directly or indirectly, from S. scabiei through a recent HGT event.

A previous phylogenetic analysis of Streptomyces and its relatives based on whole-genome sequences demonstrated that single-gene phylogenies were poorly resolved or even misleading, and the genome-based phylogeny provided a notably approved resolution (Alam et al. 2010). S. scabiei was

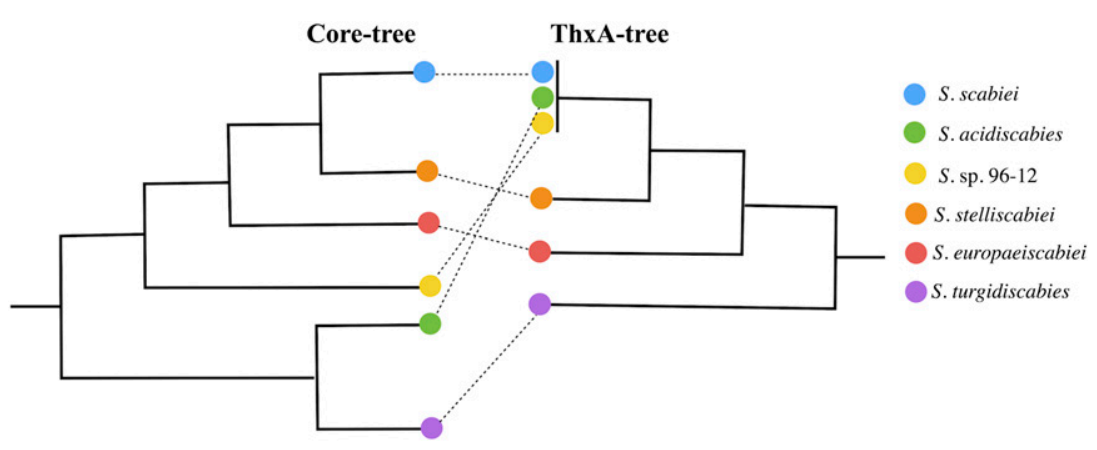

Fig. 4. Comparison of phylogenetic relationships of plant-pathogenic Streptomyces isolates derived from the core genome (left) and the thaxtomin A biosynthesis cluster (right). 


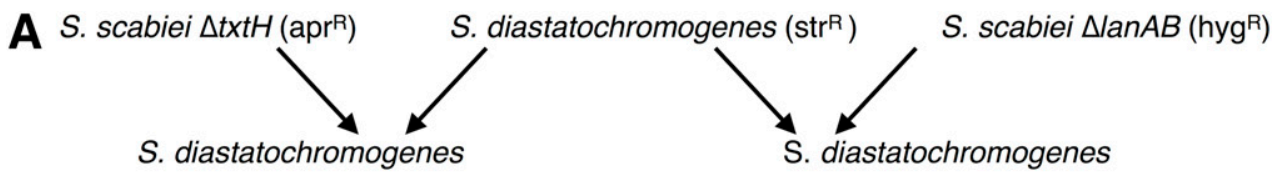

$$
\Delta t x t H\left(\operatorname{apr} r^{\mathrm{R}}, \operatorname{str}^{\mathrm{R}}\right)
$$

$\triangle \operatorname{lan} A B\left(\right.$ hyg $^{\mathrm{R}}$, str $\left.^{\mathrm{R}}\right)$

B s. diastatochromogenes S. scabiei left PAI junction S. scabiei internal PAI junction S. scabiei right PAI junction

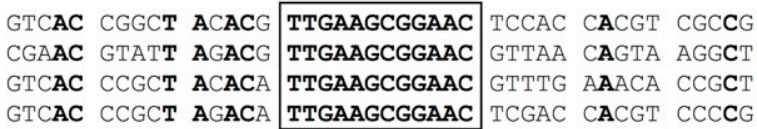

C

S. scabiei chromosome

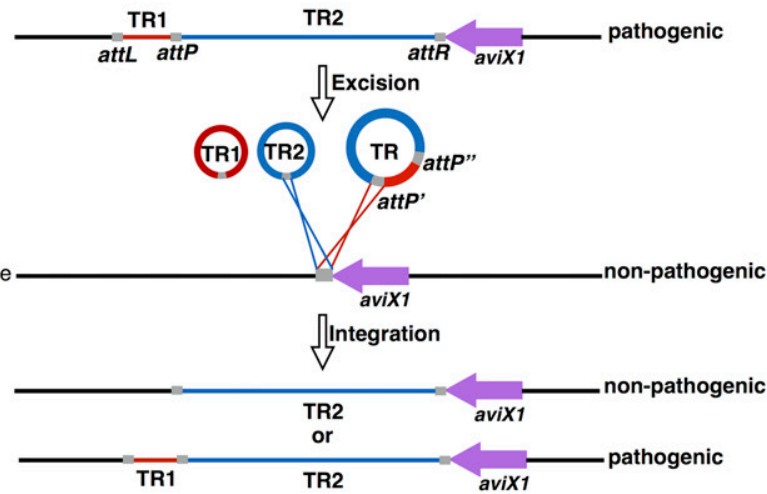

D

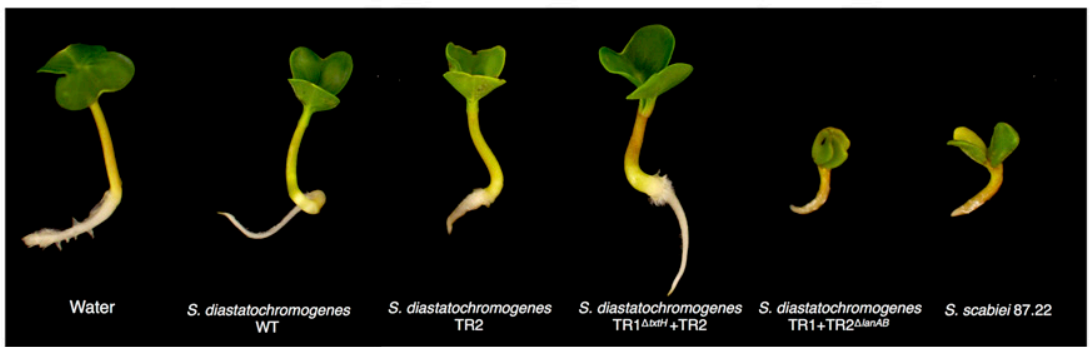

E

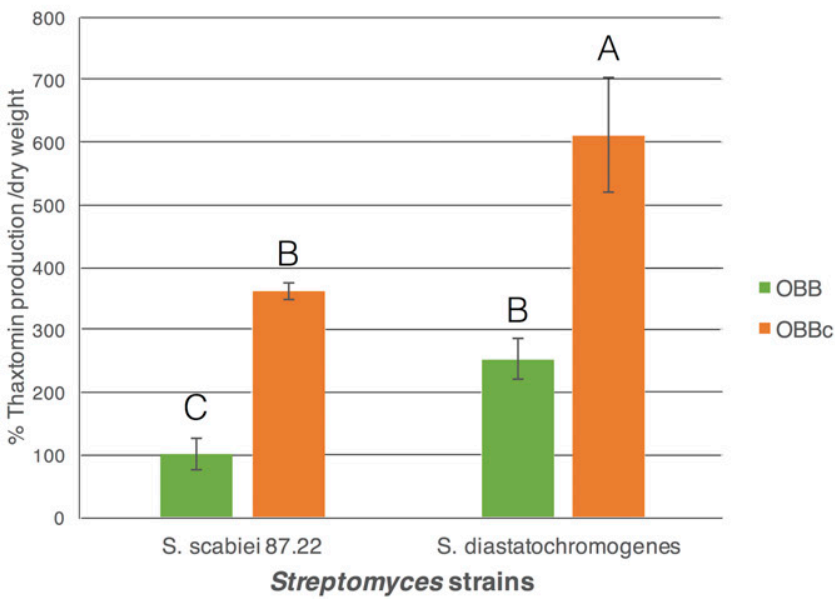

Fig. 5. Excision and site-specific integration of the Streptomyces scabiei toxicogenic region (TR) into the S. diastatochromogenes chromosome. A, Schematic representation of the mating experiments between $S$. scabiei 87-22 deletion mutants and S. diastatochromogenes. B, The pathogenicity island integrates site-specifically into the attachment (att) site located at the $3^{\prime}$ end of the aviX1 gene. C, Cartoon illustrating the three excision forms and two site-specific integration forms of S. scabiei TR; TR1 alone, TR2 alone, and the whole TR can excise from the $S$. scabiei chromosome, but only TR2 alone and the whole TR can integrate into the S. diastatochromogenes chromosome (The entire TR integration can use either $a t t P^{\prime}$ and $a t t P^{\prime}$, but our analysis only detected the integration using the $a t t P$ ' site that resulted from the recombination between att $L$ and $a t t R)$. D, Surface-sterilized newly germinated radish seedlings (six per treatment) were inoculated with mycelia from tryptic soy broth-grown cultures of $S$. diastatochromogenes transconjugants. Seedlings treated with S. scabiei 87-22, S. diastatochromogenes wild type (WT), or with water were used as controls. Plants were incubated at room temperature under a 12-h photoperiod for 4 days. Representative plants for each treatment are shown. E, Production of thaxtomin A by $S$. diastatochromogenes transconjugants that acquired the entire $\mathrm{TR}^{\triangle l a n A B}$ in oat bran broth $(\mathrm{OBB})$ medium and $\mathrm{OBB}$ medium supplemented with $1 \%$ of cellobiose $(\mathrm{OBBc})$. Isolates were grown in triplicate, in $\mathrm{OBB}$ or $\mathrm{OBBc}$ medium, for 6 days at $28^{\circ} \mathrm{C}$. The average production for each strain, normalized using dried cell weights, is shown relative to S. scabiei 87-22 in OBB (100\%); errors bars represent the standard deviation from the mean. Letters represent results of a one-way analysis of variance with Tukey's honestly significantly different test; bars not sharing letters are significantly different at $P<0.05$. 
previously considered to be most closely related to the Diastatochromogenes group (Elesawy and Szabo 1979; Healy and Lambert 1991). However, our analysis of the core genomebased phylogeny and in silico genome similarity analyses strongly suggested that $S$. diastatochromogenes is only distantly related to $S$. scabiei. These results highlight the advantages of genome sequence-based classification in the delineation of taxonomically challenging genera.

Despite being only distantly related to $S$. scabiei, S. diastatochromogenes is still able to acquire the pathogenicity phenotype upon the acqusition of the $S$. scabiei PAI. However, very few new plant-pathogenic Streptomyces species have been described since 1990 (Loria et al. 2008). The rarity of the pathogenicity phenotype within Streptomyces indicates that either PAI mobilization and stable integration is very infrequent in nature or that only a small proportion of recipients can express the pathogenicity phenotype upon acquisition of the PAI. Alternatively, there may be other genetic elements responsible for viability and reproduction inside plant tissue that have yet to be discovered but are required for pathogenicity in nature. It is important to note that, among characterized pathogens, only S. scabiei and Streptomyces sp. strain 96-12 harbor the TR2 region, which is prerequisite for the mobilization of the thaxtomin-containing TR1. The TR1 regions may have become fixed in those pathogens lacking TR2. The ThxA biosynthetic clusters of S. stelliscabiei and S. europaescabiei are distantly related to those of $S$. scabiei, S. acidiscabies, and Streptomyces sp. strain 96-12. We also observed significant genetic diversity among different $S$. europaescabiei isolates. It is possible that the ThxA biosynthetic clusters of $S$. europaescabiei and $S$. stelliscabiei have been fixed and genetic variation has accumulated after the TR fixation. However, the mobility of TRs in S. europaescabiei and S. stelliscabiei requires further investigation.

Our comparative genomic analysis of PAIs revealed their diverse evolutionary paths and highlighted the central role of ThxA in plant pathogenesis. S. scabiei is thought to be the most ancient scab-causing Streptomyces species (Bukhalid et al. 2002). Furthermore, the presence of the TR2 region is rare among pathogens. It may be that TR1 and TR2 were cotransferred into the last common ancestor of S. scabiei, S. europaescabiei, and $S$. stelliscabiei. Subsequently, the TR2 region was lost in S. europaescabiei, leading to the fixation of the TR1 region in its genome. In contrast, the TR2 region retained in S. scabiei has been a driving force in the mobilization of TR1 to otherwisenonpathogenic species, such as $S$. acidiscabies and Streptomyces sp. strain 96-12. Another possible scenario is that the TR1 region itself was transferred to the last common ancestor of S. scabiei, $S$. europaescabiei, and $S$. stelliscabiei via mechanisms independent of the TR2 region. S. scabiei may have acquired the TR2 region subsequently and, with it, the ability to transfer the TR1 region to other species, leading to the birth of novel pathogenic Streptomyces species. Due to the fragmentation of the S. stelliscabiei draft assembly, we failed to identify the complete TR2 region. A high-quality genome assembly of S. stelliscabiei is required for reconstructing the evolution history of its TR.

Inaccurate-identification of Streptomyces pathogens may have underestimated the number of pathogenic species. During the last two decades, 16S rRNA gene sequencing has been widely used for bacterial identification. However, insufficient variations among the 16S rRNA gene sequences of Streptomyces spp. result in limited taxonomic resolution (Labeda 2011; Labeda et al. 2012). Many scab-causing Streptomyces isolates were identified as $S$. scabiei, based on $16 \mathrm{~S}$ rRNA sequences; however, increased phylogenetic resolution indicates that some should be classified as other species. For example, S. europaeiscabiei NCPPB 4086 was classified as S. scabiei NCPPB 4086 when deposited in GenBank, but our study indicates it belongs to the $S$. europaeiscabiei species. Another example is Streptomyces sp. strain 96-12, which was also originally named $S$. scabiei $96-12$ by our group, because its $16 \mathrm{~S}$ rRNA is $99 \%$ identical to S. scabiei 87-22. Furthermore, Chapleau et al. (2016) reported that, of the ten S. scabiei isolates analyzed in their study, seven lacked TR2; however, here, we show that all of the verified S. scabiei isolates contain TR2. We suspect that the $S$. scabiei isolates lacking TR2 tested by Chapleau et al. (2016) actually belong to other Streptomyces species. Taken together, our results suggest that more pathogenic species exist in the natural environment than previously thought. Recently developed genome-based tools (Auch et al. 2010a; Richter and Rosselló-Móra 2009) will facilitate the accurate identification of these novel species in the future.

In summary, the results of this study revealed the horizontal transfer of the PAIs of phytopathogenic Streptomyces species and inferred its role in the emergence of new pathogenic species. Our results also highlight the notion that the current classification of only approximately ten species of Streptomyces as phytopathogens is likely due to limitations in traditional bacterial classification methods such as $16 \mathrm{~S}$ rRNA. With the increasing availability of genome sequences, genome-based tools will facilitate the accurate identification of species, reveal the existence of new species, and provide us with a more comprehensive picture of the origin and evolution of pathogenicity in the Streptomyces genus.

\section{MATERIALS AND METHODS}

\section{Bacterial strains and culture conditions.}

Escherichia coli strains were cultured in Luria-Bertani medium at $37^{\circ} \mathrm{C}$. Streptomyces strains were cultured at $28^{\circ} \mathrm{C}$ on the international Streptomyces project medium 4 agar medium or in tryptic soy broth (TSB) (BD Biosciences). All liquid cultures were shaken at $250 \mathrm{rpm}$. When required, media were supplemented with one or more of the following antibiotics at the indicated final concentrations $(\mu \mathrm{g} / \mathrm{ml})$ : hygromycin B (100), apramycin (50), kanamycin (50), chloramphenicol (25), or nalidixic acid (50). All $S$. scabiei 87-22 deletion mutants used in this study were generated by intergeneric conjugation from the nonmethylating E. coli strain ET12567/pUZ8002. Strains and plasmids used in this study are described in Supplementary Table S4.

\section{Virulence bioassays.}

To assess the virulence phenotype of different isolates, an in vitro radish seedling bioassay was performed as described previously (Bignell et al. 2010b), except that 'German Giant' seeds (Burpee) were used instead of the 'Burpee White' variety. For each strain tested, mycelial suspensions were used to inoculate six newly germinated radish seedlings of similar size. The assay was performed in triplicate with three different biological replicates each.

\section{Analysis of ThxA production.}

Plant-pathogenic Streptomyces strains were cultured in TSB for 48 to $72 \mathrm{~h}$. Mycelia were then pelleted by centrifugation, were washed twice with sterile water, and were resuspended in sterile water to obtain an optical density at $600 \mathrm{~nm}$ of 1.0. The mycelial suspension solution $(400 \mu \mathrm{l})$ was then used to inoculate $3 \times 50 \mathrm{ml}$ of OBB medium or OBBc in $250-\mathrm{ml}$ flasks. After incubation for 6 days at $28^{\circ} \mathrm{C}$ with shaking $(250 \mathrm{rpm})$, ThxA was purified from the culture supernatants and was analyzed by HPLC, as described previously (Johnson et al. 2007). All experiments were repeated at least three times using different biological replicates of the Streptomyces strains, with three technical repeats per strain. The pellets of the culture 
samples were dried and weighed as a measure of bacterial growth. The data for S. scabiei $87-22$ was normalized to $100 \%$. The data for all pathogenic isolates were square root-transformed to improve homogeneity of variance. Transformed data were subjected to one-way analysis of variance using SAS 9.4 (SAS Institute), and means were compared based on Tukey's honestly significantly difference test at $P<0.05$.

\section{Genome sequencing and assembly.}

We sequenced the genomes using the Illumina-HiSeq 2500 paired-end technology. For each genome, the SPAdes version 3.5.0 assembly algorithm (Bankevich et al. 2012) was used to perform genome assembly. Gene-finding and annotation were achieved using the rapid annotation using subsystems technology (RAST) server (Aziz et al. 2008).

\section{Ortholog retrieval and single-copy cluster search.}

To build the rooted phylogenetic tree, Streptacidiphilus albus JL83 and Kitasatospora setae NBRC 14216 were selected as the outgroups. Genes from 111 Streptomyces genomes and the two outgroups were clustered into ortholog groups using OrthoMCL v1.4 (Li et al. 2003).

We first conducted an all-against-all protein sequence comparison of the 113 strains using BLASTP and defined putative pairs of orthologs based on reciprocal BLASTP. Subsequently, the reciprocal BLASTP values were converted to a normalized similarity matrix that was analyzed by a Markov cluster algorithm (Enright et al. 2002), which yielded a set of clusters of homologs. OrthoMCL was run with a BLAST E-value cut-off of $1 \mathrm{e}^{-5}$, a minimum aligned sequence length coverage of $50 \%$ of the query sequence, and an inflation index of 1.5. Ortholog groups with a single copy per genome were selected as potential phylogenetic markers.

\section{Gene alignment and filtering criteria.}

To minimize inclusion of alignments of low quality in the analysis, we used various filtering criteria. Alignments for each of the single-copy orthologs were generated at the amino acid level using Probalign v1.1 (Roshan and Livesay 2006) and were then back-translated to DNA sequences, based on the original genomic sequence. Subsequently, alignment columns with a posterior probability $<0.6$ were removed (Lefébure and Stanhope 2009). Alignments made after removing all ambiguous positions that were less than $50 \%$ of the original alignment length were excluded (Lefébure and Stanhope 2009).

\section{ML phylogeny estimation.}

The gene alignment was used for ML inference on the RAxML webserver (https://www.phylo.org/portal2/) (Stamatakis et al. 2008) with the GTR+G substitution model, including an estimation of invariable sites. For inferring tree robustness, 1,000 bootstrap replicates were computed.

\section{ANI and isDDH estimation.}

The ANI analysis of whole-genome data was performed using the method proposed by Richter and Rosselló-Móra (Richter and Rosselló-Móra 2009). Pair-wise ANI values were obtained from nucmer (Delcher et al. 2002) with the script calculate_ani.py (https:/github.com/widdowquinn/scripts/blob/ master/bioinformatics/calculate_ani.py).

The isDDH was estimated using the genome-to-genome distance calculator (GGDC) (Auch et al. 2010b; Meier-Kolthoff et al. 2013). The contig files were uploaded to the GGDC 2.0 Web server (http://ggdc.dsmz.de/distcalc2.php), where isDDH calculations were performed using Formula 2 alone. We used the point estimates plus the $95 \%$ model-based confidence intervals as the isDDH estimates.
Insertion of antibiotic resistance markers in the PAI.

Insertion of antibiotic resistance markers into the TR1 or TR2 region of S. scabiei 87-22 was accomplished by replacing txtH (SCAB_31771) with the apr ${ }^{\mathrm{R}}$ gene or replacing the lantibiotic biosynthetic genes lanA (SCAB_32021) and lanB (SCAB_32031) with the hyg ${ }^{\mathrm{R}}$ gene using the redirect PCR targeting system (Gust et al. 2003).

Cosmid 1989 (harboring the $t x t H$ gene) or cosmid 2757 (harboring the lantibiotic biosynthesis genes) was introduced to the E. coli BW25113 strain harboring the arabinose-inducible $\lambda$ red expression plasmid pIJ790 (aac[3]IV + oriT) or pIJ10700 (hyg + oriT). The deletion cassette for $t x t H$ was PCR amplified using pIJ773 as the template and primers DRB201 and DRB202; the deletion cassette for lanA and lanB was PCR amplified using pIJ10700 as the template and primers DRB431 and DRB432. The gel-purified deletion cassettes were electroporated into the $E$. coli BW25113 cells that contained either cosmid 1989 or cosmid 2757 and which had been induced with arabinose $(20 \mathrm{mM}$ final concentration). The $\triangle t x t H$ and $\triangle l a n A B$ mutant cosmids were then isolated and were confirmed by PCR and sequencing. Following transformation of each cosmid into the nonmethylating $E$. coli ET12567 strain (MacNeil et al. 1992), which contains the plasmid pUZ8002 as a driver for transfer (Bierman et al. 1992), the cosmids were conjugated into $S$. scabiei $87-22$ on soy flour mannitol (SFM) agar (Kieser et al. 2000). Resulting exconjugants for $\Delta t x t H$ were selected for resistance to apramycin $\left(\operatorname{apr}^{\mathrm{R}}\right)$ and sensitive to kanamycin, and exconjugants for $\triangle \operatorname{lan} A B$ were selected for resistance to hygromycin $B\left(\right.$ hyg $\left.^{\mathrm{R}}\right)$ and sensitive to kanamycin. The mutant strains were confirmed using PCR and were stored as spore stocks in $20 \% \mathrm{vol} / \mathrm{vol}$ glycerol at $-80^{\circ} \mathrm{C}$.

\section{Selection of transconjugant strains.}

Conjugal mating was carried out using either $S$. scabiei 87-22 $\Delta$ txt $H\left(\operatorname{apr}^{\mathrm{R}}\right)$ or S. scabiei 87-22 $\Delta$ lanAB $\left(\right.$ hyg $\left.^{\mathrm{R}}\right)$ as a PAI donor strain and naturally $\operatorname{str}^{\mathrm{R}} S$. diastatochromogenes as the recipient strain. Strains were cocultured on SFM plates (Kieser et al. 2000) for 2 days at $28^{\circ} \mathrm{C}$. A soft nutrient agar overlay containing (per milliliter) $20 \mu \mathrm{g}$ of streptomycin and $50 \mu \mathrm{g}$ of apramycin or $100 \mu \mathrm{g}$ of hygromycin B (final concentration) was used to select for $\operatorname{apr}^{R} \operatorname{str}^{R}$ or hyg ${ }^{R} \operatorname{str}^{R}$ transconjugants. Qualitative PCR assays were conducted to detect the integration of $S$. scabiei TR into the chromosome of $S$. diastatochromogenes. Primers $\mathrm{a}+\mathrm{b}$, flanking the att site located at the $3^{\prime}$ end of the $S$. diastatochromogenes aviX1 gene, were used to detect the site-specific integration of TR in aviX1 (final PCR products for S. diastatochromogenes wild type, 724 bp). Primers e $+\mathrm{f}$ (final PCR products, $731 \mathrm{bp}$ ) were used to amplify the chromosomal junction formed by integration of TR or TR2 into $S$. diastatochromogenes aviX1. Primers $\mathrm{c}+\mathrm{d}$, which are located within the integrase gene (SCAB_31871) of TR2, were used to confirm TR2 integration (final PCR products, $656 \mathrm{bp}$ ). Primers $\mathrm{g}+\mathrm{h}$, flanking $t x t H$ (SCAB_31771), were used to confirm TR1 integration (final PCR products, $450 \mathrm{bp}$ ). All PCR products were sequenced.

Frequency of TR transfer was estimated by replicate plating $(n=3)$, using an approximately 1:1 ratio of donor (S. scabiei 87-22 $\triangle$ lan $A B$ ) to recipient (S. diastatochromogenes) spores on SFM medium (approximately $10^{5}$ to $10^{6}$ CFU per 100 -mm Petri dish). Frequency of TR integration was estimated based on the number of hyg ${ }^{\mathrm{R}}$ and $\mathrm{str}^{\mathrm{R}}$ colonies.

\section{ACKNOWLEDGMENTS}

This work was partially supported by the Agriculture and Food Research Initiative Competitive Grants Program (grant 2010-65110-20416 from the United States Department of Agriculture's National Institute of Food and Agriculture to R. Loria), University of Florida startup funds, and the Ralph E. Powe Junior Faculty Enhancement Award (to Y. Ding). 


\section{LITERATURE CITED}

Achtman, M., and Wagner, M. 2008. Microbial diversity and the genetic nature of microbial species. Nat. Rev. Microbiol. 6:431-440.

Alam, M. T., Merlo, M. E., Takano, E., and Breitling, R. 2010. Genomebased phylogenetic analysis of Streptomyces and its relatives. Mol. Phylogenet. Evol. 54:763-772.

Auch, A. F., von Jan, M., Klenk, H. P., and Göker, M. 2010b. Digital DNADNA hybridization for microbial species delineation by means of genometo-genome sequence comparison. Stand. Genomic Sci. 2:117-134.

Auch, A. F., Klenk, H.-P., and Göker, M. 2010a. Standard operating procedure for calculating genome-to-genome distances based on highscoring segment pairs. Stand. Genomic Sci. 2:142-148.

Aziz, R. K., Bartels, D., Best, A. A., DeJongh, M., Disz, T., Edwards, R. A., Formsma, K., Gerdes, S., Glass, E. M., Kubal, M., Meyer, F., Olsen, G. J., Olson, R., Osterman, A. L., Overbeek, R. A., McNeil, L. K., Paarmann, D., Paczian, T., Parrello, B., Pusch, G. D., Reich, C., Stevens, R., Vassieva, O., Vonstein, V., Wilke, A., and Zagnitko, O. 2008. The RAST Server: Rapid annotations using subsystems technology. BMC Genomics 9:75.

Bankevich, A., Nurk, S., Antipov, D., Gurevich, A. A., Dvorkin, M., Kulikov, A. S., Lesin, V. M., Nikolenko, S. I., Pham, S., Prjibelski, A. D., Pyshkin, A. V., Sirotkin, A. V., Vyahhi, N., Tesler, G., Alekseyev, M. A., and Pevzner, P. A. 2012. SPAdes: A new genome assembly algorithm and its applications to single-cell sequencing. J. Comput. Biol. 19: 455-477.

Bentley, S. D., Chater, K. F., Cerdeño-Tárraga, A.-M., Challis, G. L., Thomson, N. R., James, K. D., Harris, D. E., Quail, M. A., Kieser, H., Harper, D., Bateman, A., Brown, S., Chandra, G., Chen, C. W., Collins, M., Cronin, A., Fraser, A., Goble, A., Hidalgo, J., Hornsby, T., Howarth, S., Huang, C. H., Kieser, T., Larke, L., Murphy, L., Oliver, K., O’Neil, S., Rabbinowitsch, E., Rajandream, M.-A., Rutherford, K., Rutter, S., Seeger, K., Saunders, D., Sharp, S., Squares, R., Squares, S., Taylor, K. Warren, T., Wietzorrek, A., Woodward, J., Barrell, B. G., Parkhill, J., and Hopwood, D. A. 2002. Complete genome sequence of the model actinomycete Streptomyces coelicolor A3(2). Nature 417:141-147.

Bierman, M., Logan, R., O’Brien, K., Seno, E. T., Rao, R. N., and Schoner, B. E. 1992. Plasmid cloning vectors for the conjugal transfer of DNA from Escherichia coli to Streptomyces spp. Gene 116:43-49.

Bignell, D. R. D., Fyans, J. K., and Cheng, Z. 2014. Phytotoxins produced by plant-pathogenic Streptomyces species. J. Appl. Microbiol. 116: 223-235.

Bignell, D. R. D., Huguet-Tapia, J. C., Joshi, M. V., Pettis, G. S., and Loria, R. 2010a. What does it take to be a plant pathogen: Genomic insights from Streptomyces species. Antonie van Leeuwenhoek 98:179-194.

Bignell, D. R. D., Seipke, R. F., Huguet-Tapia, J. C., Chambers, A. H., Parry, R. J., and Loria, R. 2010b. Streptomyces scabies 87-22 contains a coronafacic acid-like biosynthetic cluster that contributes to plant-microbe interactions. Mol. Plant-Microbe Interact. 23:161-175.

Bukhalid, R. A., Takeuchi, T., Labeda, D., and Loria, R. 2002. Horizontal transfer of the plant virulence gene, necl, and flanking sequences among genetically distinct Streptomyces strains in the Diastatochromogenes cluster. Appl. Environ. Microbiol. 68:738-744.

Chapleau, M., Guertin, J. F., Farrokhi, A., Lerat, S., Burrus, V., and Beaulieu, C. 2016. Identification of genetic and environmental factors stimulating excision from Streptomyces scabiei chromosome of the toxicogenic region responsible for pathogenicity. Mol. Plant Pathol. 17:501-509.

Colston, S. M., Fullmer, M. S., Beka, L., Lamy, B., Gogarten, J. P., and Graf, J. 2014. Bioinformatic genome comparisons for taxonomic and phylogenetic assignments using Aeromonas as a test case. MBio 5: e02136-14.

Delcher, A. L., Phillippy, A., Carlton, J., and Salzberg, S. L. 2002. Fast algorithms for large-scale genome alignment and comparison. Nucleic Acids Res. 30:2478-2483.

Elesawy, A. A., and Szabo, M. 1979. Isolation and characterization of Streptomyces scabies strains from scab lesions of potato tubers. Designation of the neotype strain of Streptomyces scabies. Acta Microbiol. Acad. Sci. Hung. 26:311-320.

Enright, A. J., Van Dongen, S., and Ouzounis, C. A. 2002. An efficient algorithm for large-scale detection of protein families. Nucleic Acids Res. 30:1575-1584

Francis, I. M., Jourdan, S., Fanara, S., Loria, R., and Rigali, S. 2015. The cellobiose sensor CebR is the gatekeeper of Streptomyces scabies pathogenicity. MBio 6:e02018-14.

Fry, B. A., and Loria, R. 2002. Thaxtomin A: Evidence for a plant cell wall target. Physiol. Mol. Plant Pathol. 60:1-8.

Goris, J., Konstantinidis, K. T., Klappenbach, J. A., Coenye, T., Vandamme, P., and Tiedje, J. M. 2007. DNA-DNA hybridization values and their relationship to whole-genome sequence similarities. Int. J. Syst. Evol. Microbiol. 57:81-91.

Guan, D., Grau, B. L., Clark, C. A., Taylor, C. M., Loria, R., and Pettis, G. S. 2012. Evidence that thaxtomin C is a pathogenicity determinant of Streptomyces ipomoeae, the causative agent of Streptomyces soil rot disease of sweet potato. Mol. Plant-Microbe Interact. 25:393-401.

Guo, Y., Zheng, W., Rong, X., and Huang, Y. 2008. A multilocus phylogeny of the Streptomyces griseus 16S rRNA gene clade: Use of multilocus sequence analysis for streptomycete systematics. Int. J. Syst. Evol. Microbiol. 58:149-159.

Gust, B., Challis, G. L., Fowler, K., Kieser, T., and Chater, K. F. 2003. PCRtargeted Streptomyces gene replacement identifies a protein domain needed for biosynthesis of the sesquiterpene soil odor geosmin. Proc. Natl. Acad. Sci. U.S.A. 100:1541-1546.

Healy, F. G., Krasnoff, S. B., Wach, M., Gibson, D. M., and Loria, R. 2002. Involvement of a cytochrome P450 monooxygenase in thaxtomin A biosynthesis by Streptomyces acidiscabies. J. Bacteriol. 184:2019-2029.

Healy, F. G., and Lambert, D. H. 1991. Relationships among Streptomyces spp. causing potato scab. Int. J. Syst. Evol. Microbiol. 41:479-482.

Herbst, D. A., Boll, B., Zocher, G., Stehle, T., and Heide, L. 2013. Structural basis of the interaction of MbtH-like proteins, putative regulators of nonribosomal peptide biosynthesis, with adenylating enzymes. J. Biol. Chem. 288:1991-2003.

Huguet-Tapia, J. C., Badger, J. H., Loria, R., and Pettis, G. S. 2011. Streptomyces turgidiscabies Car8 contains a modular pathogenicity island that shares virulence genes with other actinobacterial plant pathogens. Plasmid 65:118-124.

Huguet-Tapia, J. C., Bignell, D. R. D., and Loria, R. 2014. Characterization of the integration and modular excision of the integrative conjugative element PAISt in Streptomyces turgidiscabies Car8. PLoS One 9:e99345.

Huguet-Tapia, J. C., Lefébure, T., Badger, J. H., Guan, D., Pettis, G. S., Stanhope, M. J., and Loria, R. 2016. Genome content and phylogenomics reveal both ancestral and lateral evolutionary pathways in plant-pathogenic Streptomyces species. Appl. Environ. Microbiol. 82: 2146-2155.

Huguet-Tapia, J. C., and Loria, R. 2012. Draft genome sequence of Streptomyces acidiscabies 84-104, an emergent plant pathogen. J. Bacteriol. 194:1847.

Johnson, E. G., Joshi, M. V., Gibson, D. M., and Loria, R. 2007. Cellooligosaccharides released from host plants induce pathogenicity in scabcausing Streptomyces species. Physiol. Mol. Plant Pathol. 71:18-25.

Joshi, M., Rong, X., Moll, S., Kers, J., Franco, C., and Loria, R. 2007. Streptomyces turgidiscabies secretes a novel virulence protein, Nec1, which facilitates infection. Mol. Plant-Microbe Interact. 20:599-608.

Joshi, M. V., and Loria, R. 2007. Streptomyces turgidiscabies possesses a functional cytokinin biosynthetic pathway and produces leafy galls. Mol. Plant-Microbe Interact. 20:751-758.

Kers, J. A., Cameron, K. D., Joshi, M. V., Bukhalid, R. A., Morello, J. E., Wach, M. J., Gibson, D. M., and Loria, R. 2005. A large, mobile pathogenicity island confers plant pathogenicity on Streptomyces species. Mol. Microbiol. 55:1025-1033.

Kieser, T., Bibb, M. J., Buttner, M. J., Chater, K. F., and Hopwood, D. A. 2000. Practical Streptomyces Genetics. John Innes Foundation, Norwich, U. K.

King, R. R., Lawrence, C. H., and Gray, J. A. 2001. Herbicidal properties of the thaxtomin group of phytotoxins. J. Agric. Food Chem. 49:22982301

Kreuze, J. F., Suomalainen, S., Paulin, L., and Valkonen, J. P. T. 1999. Phylogenetic analysis of 16S rRNA genes and PCR analysis of the necl gene from Streptomyces spp. causing common scab, pitted scab, and netted scab in Finland. Phytopathology 89:462-469.

Labeda, D. P. 2011. Multilocus sequence analysis of phytopathogenic species of the genus Streptomyces. Int. J. Syst. Evol. Microbiol. 61: 2525-2531.

Labeda, D. P., Goodfellow, M., Brown, R., Ward, A. C., Lanoot, B., Vanncanneyt, M., Swings, J., Kim, S. B., Liu, Z., Chun, J., Tamura, T., Oguchi, A., Kikuchi, T., Kikuchi, H., Nishii, T., Tsuji, K., Yamaguchi, Y., Tase, A., Takahashi, M., Sakane, T., Suzuki, K. I., and Hatano, K. 2012. Phylogenetic study of the species within the family Streptomycetaceae. Antonie van Leeuwenhoek 101:73-104.

Lefébure, T., and Stanhope, M. J. 2009. Pervasive, genome-wide positive selection leading to functional divergence in the bacterial genus Campylobacter. Genome Res. 19:1224-1232.

Lerat, S., Simao-Beaunoir, A.-M., and Beaulieu, C. 2009. Genetic and physiological determinants of Streptomyces scabies pathogenicity. Mol. Plant Pathol. 10:579-585.

Li, L., Stoeckert, C. J., Jr., and Roos, D. S. 2003. OrthoMCL: Identification of ortholog groups for eukaryotic genomes. Genome Res. 13:2178-2189. 
Loria, R., Bignell, D. R. D., Moll, S., Huguet-Tapia, J. C., Joshi, M. V., Johnson, E. G., Seipke, R. F., and Gibson, D. M. 2008. Thaxtomin biosynthesis: The path to plant pathogenicity in the genus Streptomyces. Antonie van Leeuwenhoek 94:3-10.

Loria, R., Bukhalid, R. A., Fry, B. A., and King, R. R. 1997. Plant pathogenicity in the genus Streptomyces. Plant Dis. 81:836-846.

Loria, R., Kers, J., and Joshi, M. 2006. Evolution of plant pathogenicity in Streptomyces. Annu. Rev. Phytopathol. 44:469-487.

MacNeil, D. J., Gewain, K. M., Ruby, C. L., Dezeny, G., Gibbons, P. H., and MacNeil, T. 1992. Analysis of Streptomyces avermitilis genes required for avermectin biosynthesis utilizing a novel integration vector. Gene 111:61-68.

Meier-Kolthoff, J. P., Auch, A. F., Klenk, H.-P., and Göker, M. 2013. Genome sequence-based species delimitation with confidence intervals and improved distance functions. BMC Bioinformatics 14:60.

Pertry, I., Václavíková, K., Gemrotová, M., Spíchal, L., Galuszka, P., Depuydt, S., Temmerman, W., Stes, E., De Keyser, A., Riefler, M., Biondi, S., Novák, O., Schmülling, T., Strnad, M., Tarkowski, P., Holsters, M., and Vereecke, D. 2010. Rhodococcus fascians impacts plant development through the dynamic fas-mediated production of a cytokinin mix. Mol. Plant-Microbe Interact. 23:1164-1174.

Richter, M., and Rosselló-Móra, R. 2009. Shifting the genomic gold standard for the prokaryotic species definition. Proc. Natl. Acad. Sci. U.S.A. 106:19126-19131.

Roshan, U., and Livesay, D. R. 2006. Probalign: Multiple sequence alignment using partition function posterior probabilities. Bioinformatics 22:2715-2721.
Salichos, L., and Rokas, A. 2013. Inferring ancient divergences requires genes with strong phylogenetic signals. Nature 497:327-331.

Seipke, R. F., and Loria, R. 2008. Streptomyces scabies 87-22 possesses a functional tomatinase. J. Bacteriol. 190:7684-7692.

Stamatakis, A., Hoover, P., and Rougemont, J. 2008. A rapid bootstrap algorithm for the RAxML Web servers. Syst. Biol. 57:758-771.

Stegmann, E., Rausch, C., Stockert, S., Burkert, D., and Wohlleben, W. 2006. The small MbtH-like protein encoded by an internal gene of the balhimycin biosynthetic gene cluster is not required for glycopeptide production. FEMS (Fed. Eur. Microbiol. Soc.) Microbiol. Lett. 262: 85-92.

Wach, M. J., Krasnoff, S. B., Loria, R., and Gibson, D. M. 2007. Effect of carbohydrates on the production of thaxtomin A by Streptomyces acidiscabies. Arch. Microbiol. 188:81-88.

Wozniak, R. A. F., and Waldor, M. K. 2010. Integrative and conjugative elements: Mosaic mobile genetic elements enabling dynamic lateral gene flow. Nat. Rev. Microbiol. 8:552-563.

Zhang, Y., Fan, Q., and Loria, R. 2016. A re-evaluation of the taxonomy of phytopathogenic genera Dickeya and Pectobacterium using wholegenome sequencing data. Syst. Appl. Microbiol. 39:252-259.

Zhang, Y., and Qiu, S. 2015. Examining phylogenetic relationships of Erwinia and Pantoea species using whole genome sequence data. Antonie van Leeuwenhoek 108:1037-1046.

Zhang, Y., and Qiu, S. 2016. Phylogenomic analysis of the genus Ralstonia based on 686 single-copy genes. Antonie van Leeuwenhoek 109:71-82. 